\title{
Approximation in weighted Smirnov-Orlicz classes
}

\author{
By
}

Daniyal M. IsRAFILOV and Ramazan AKGÜN

\begin{abstract}
In this work some direct and inverse theorems of approximation theory in the weighted Smirnov-Orlicz classes, defined in the domains with a Dini-smooth boundary, are proved. In particular, a constructive characterization of the generalized Lipschitz classes $\operatorname{Lip}^{*} \alpha(M, \omega), \alpha>0$, is obtained.
\end{abstract}

\section{Introduction and main results}

Let $\Gamma \subset \mathbb{C}$ be a closed bounded rectifiable Jordan curve in the complex plane $\mathbb{C}$. $\Gamma$ separates the plane $\mathbb{C}$ into two domains $G:=\operatorname{int} \Gamma, G^{-}:=\operatorname{ext} \Gamma$. Without loss of generality we may assume $0 \in G$. Let $\mathbb{D}:=\{w \in \mathbb{C}:|w|<1\}$, $\mathbb{T}:=\partial \mathbb{D}, \mathbb{D}^{-}:=\operatorname{ext} \mathbb{T}$ and $w=\varphi(z)$ be the conformal mapping of $G^{-}$onto $\mathbb{D}^{-}$ normalized by the conditions

$$
\varphi(\infty)=\infty, \quad \lim _{z \rightarrow \infty} \varphi(z) / z>0,
$$

and let $\psi:=\varphi^{-1}$ be the inverse mapping of $\varphi$.

By $E^{p}(G), 0<p<\infty$, we denote the Smirnov class of analytic functions in $G$. Every function in $E^{p}(G), 1 \leq p<\infty$, has the non-tangential boundary values almost everywhere (a. e.) on $\Gamma$ and the boundary function belongs to Lebesgue space $L^{p}(\Gamma)[7$, p. 438].

Let $h$ be a continuous function on $[0,2 \pi]$. Its modulus of continuity is defined by

$$
\omega(t, h):=\sup \left\{\left|h\left(t_{1}\right)-h\left(t_{2}\right)\right|: t_{1}, t_{2} \in[0,2 \pi],\left|t_{1}-t_{2}\right| \leq t\right\}, \quad t \geq 0 .
$$

The function $h$ is called Dini-continuous if

$$
\int_{0}^{\pi} \frac{\omega(t, h)}{t} d t<\infty .
$$

2000 Mathematics Subject Classification(s). 30E10, 41A10, 41A25, 46E30. Received February 24, 2006 
The curve $\Gamma$ is called Dini-smooth if it has a parametrization

$$
\Gamma: \varphi_{0}(\tau), \quad 0 \leq \tau \leq 2 \pi
$$

such that $\varphi_{0}^{\prime}(\tau)$ is Dini-continuous and $\varphi_{0}^{\prime}(\tau) \neq 0$ [22, p. 48].

When $\Gamma$ is Dini-smooth, [24] asserts that

$$
\begin{array}{ll}
0<c_{1} \leq\left|\psi^{\prime}(w)\right| \leq c_{2}, & |w| \geq 1, \\
0<c_{3} \leq\left|\varphi^{\prime}(z)\right| \leq c_{4}, & z \in G^{-},
\end{array}
$$

for some constants $c_{1}, c_{2}$ and $c_{3}, c_{4}$ independent of $w$ and $z$, respectively.

A continuous and convex function $M:[0, \infty) \rightarrow[0, \infty)$ which satisfies the conditions

$$
\begin{gathered}
M(0)=0 ; \quad M(x)>0 \quad \text { for } x>0 ; \\
\lim _{x \rightarrow 0}(M(x) / x)=0 ; \quad \lim _{x \rightarrow \infty}(M(x) / x)=\infty,
\end{gathered}
$$

is called an $N$-function.

The complementary $N$-function to $M$ is defined by

$$
N(y):=\max _{x \geq 0}(x y-M(x)), \quad y \geq 0 .
$$

We denote by $L_{M}(\Gamma)$ the linear space of Lebesgue measurable functions $f$ : $\Gamma \rightarrow \mathbb{C}$ satisfying the condition

$$
\int_{\Gamma} M[\alpha|f(z)|]|d z|<\infty
$$

for some $\alpha>0$.

The space $L_{M}(\Gamma)$ becomes a Banach space with the Luxemburg norm

$$
\|f\|_{L_{(M)}(\Gamma)}:=\inf \{\tau>0: \rho(f / \tau ; M) \leq 1\},
$$

and also with the Orlicz norm

$$
\|f\|_{L_{M}(\Gamma)}:=\sup \left\{\int_{\Gamma}|f(z) g(z)||d z|: g \in L_{N}(\Gamma) ; \rho(g ; N) \leq 1\right\},
$$

where $N$ is the complementary $N$-function to $M$ and

$$
\rho(g ; N):=\int_{\Gamma} N[|g(z)|]|d z| .
$$

The Banach space $L_{M}(\Gamma)$ is called Orlicz space.

A function $\omega$ is called a weight on $\Gamma$ if $\omega: \Gamma \rightarrow[0, \infty]$ is measurable and $\omega^{-1}(\{0, \infty\})$ has measure zero (with respect to Lebesgue measure). 
The class of measurable functions $f$ defined on $\Gamma$ and satisfying the condition $\omega f \in L_{M}(\Gamma)$ is called weighted Orlicz space $L_{M}(\Gamma, \omega)$ with the norm

$$
\|f\|_{L_{M}(\Gamma, \omega)}:=\|f \omega\|_{L_{M}(\Gamma)} .
$$

For $z \in \Gamma$ and $\epsilon>0$ let $\Gamma(z, \epsilon)$ denotes the portion of $\Gamma$ contained in the open disc of radius $\epsilon$ and centered at $z$, i.e. $\Gamma(z, \epsilon):=\{t \in \Gamma:|t-z|<\epsilon\}$.

For fixed $p \in(1, \infty)$, we define $q \in(1, \infty)$ by $p^{-1}+q^{-1}=1$. The set of all weights $\omega: \Gamma \rightarrow[0, \infty]$ satisfying the relation

$$
\sup _{t \in \Gamma} \sup _{\epsilon>0}\left(\frac{1}{\epsilon} \int_{\Gamma(z, \epsilon)} \omega(\tau)^{p}|d \tau|\right)^{1 / p}\left(\frac{1}{\epsilon} \int_{\Gamma(z, \epsilon)} \omega(\tau)^{-q}|d \tau|\right)^{1 / q}<\infty
$$

is denoted by $A_{p}(\Gamma)$.

We denote by $L^{p}(\Gamma, \omega)$ the set of all measurable functions $f: \Gamma \rightarrow \mathbb{C}$ such that $|f| \omega \in L^{p}(\Gamma), 1<p<\infty$.

Let $M^{-1}:[0, \infty) \rightarrow[0, \infty)$ be the inverse function of the $N$-function $M$. The lower and upper indices $\alpha_{M}, \beta_{M}[3$, p. 350]

$$
\alpha_{M}:=\lim _{x \rightarrow 0} \frac{\log \varrho(x)}{\log x}, \quad \beta_{M}:=\lim _{x \rightarrow \infty} \frac{\log \varrho(x)}{\log x}
$$

of the function

$$
\varrho:(0, \infty) \rightarrow(0, \infty], \quad \varrho(x):=\limsup _{y \rightarrow \infty} \frac{M^{-1}(y)}{M^{-1}(y / x)}, \quad x \in(0, \infty),
$$

first considered by W. Matuszewska and W. Orlicz [20], are called the Boyd indices of the Orlicz space $L_{M}(\Gamma)$. It is well known that $0 \leq \alpha_{M} \leq \beta_{M} \leq 1$. For this and other properties of Boyd indices of Orlicz spaces we refer to [19].

The indices $\alpha_{M}, \beta_{M}$ are called nontrivial if $0<\alpha_{M}$ and $\beta_{M}<1$.

Definition 1. For a weight $\omega$ on $\Gamma$ we denote by $E_{M}(G, \omega)$ the subclass of analytic functions of $E^{1}(G)$ whose boundary value functions belong to weighted Orlicz space $L_{M}(\Gamma, \omega)$.

The weighted Smirnov-Orlicz class $E_{M}(G, \omega)$ is a generalization of the Smirnov class $E^{p}(G)$. In particular, if $M(x):=x^{p}, 1<p<\infty$, then the weighted Smirnov-Orlicz class $E_{M}(G, \omega)$ coincides with the weighted Smirnov class $E^{p}(G, \omega)$; if $\omega:=1$, then $E_{M}(G, \omega)$ coincides with the Smirnov-Orlicz class $E_{M}(G)$, defined in [18].

Let $\Gamma$ be a rectifiable Jordan curve and $f \in L^{1}(\Gamma)$. The functions $f^{+}$and $f^{-}$defined by

$$
f^{+}(z)=\frac{1}{2 \pi i} \int_{\Gamma} \frac{f(\varsigma)}{\varsigma-z} d \varsigma, \quad z \in G
$$

and

$$
f^{-}(z)=\frac{1}{2 \pi i} \int_{\Gamma} \frac{f(\varsigma)}{\varsigma-z} d \varsigma, \quad z \in G^{-}
$$


are analytic in $G$ and $G^{-}$, respectively and $f^{-}(\infty)=0$.

For $g \in L_{M}(\mathbb{T}, \omega)$ we set

$$
\sigma_{h}(g)(w):=\frac{1}{2 h} \int_{-h}^{h} g\left(w e^{i t}\right) d t, \quad 0<h<\pi, \quad w \in \mathbb{T} .
$$

If $\alpha_{M}$ and $\beta_{M}$ are nontrivial and $\omega \in A_{\frac{1}{\alpha_{M}}}(\mathbb{T}) \cap A_{\frac{1}{\beta_{M}}}(\mathbb{T})$, then by [14] we have

$$
\left\|\sigma_{h}(g)\right\|_{L_{M}(\mathbb{T}, \omega)} \leq c_{5}\|g\|_{L_{M}(\mathbb{T}, \omega)},
$$

and consequently $\sigma_{h}(g) \in L_{M}(\mathbb{T}, \omega)$ for any $g \in L_{M}(\mathbb{T}, \omega)$.

Definition 2. Let $\alpha_{M}$ and $\beta_{M}$ be nontrivial and $\omega \in A_{\frac{1}{\alpha_{M}}}(\mathbb{T}) \cap$ $A_{\frac{1}{\beta_{M}}}(\mathbb{T})$. The function

$$
\Omega_{M, \omega}^{r}(g, \delta):=\sup _{\substack{0<h_{i} \leq \delta \\ i=1,2, \ldots, r}}\left\|\prod_{i=1}^{r}\left(I-\sigma_{h_{i}}\right) g\right\|_{L_{M}(\mathbb{T}, \omega)}, \quad \delta>0, \quad r=1,2, \ldots
$$

is called rth modulus of smoothness of $g \in L_{M}(\mathbb{T}, \omega)$, where $I$ is the identity operator.

Note that in case of weighted Lebesgue spaces $L^{p}(\mathbb{T}, \omega)$ this definition originates from [25] (see also [10], [11], [12]).

It is easily verified that the function $\Omega_{M, \omega}(g, \cdot)$ is continuous, non-negative and satisfy

$$
\lim _{\delta \rightarrow 0} \Omega_{M, \omega}^{r}(g, \delta)=0, \quad \Omega_{M, \omega}^{r}\left(g+g_{1}, \cdot\right) \leq \Omega_{M, \omega}^{r}(g, \cdot)+\Omega_{M, \omega}^{r}\left(g_{1}, \cdot\right)
$$

for $g, g_{1} \in L_{M}(\mathbb{T}, \omega)$.

Let $\omega_{0}(w):=\omega(\psi(w))$ and $f_{0}(w):=f(\psi(w))$ for a weight $\omega$ on $\Gamma, f \in$ $L_{M}(\Gamma, \omega)$ and $w \in \mathbb{T}$. By $(1.1)$ we have $f_{0} \in L_{M}\left(\mathbb{T}, \omega_{0}\right)$ for $f \in L_{M}(\Gamma, \omega)$. Using the nontangential boundary values of $f_{0}^{+}$on $\mathbb{T}$ we define the rth modulus of smoothness of $f \in L_{M}(\Gamma, \omega)$ as

$$
\Omega_{\Gamma, M, \omega}^{r}(f, \delta):=\Omega_{M, \omega_{0}}^{r}\left(f_{0}^{+}, \delta\right), \quad \delta>0,
$$

for $r=1,2,3, \ldots$

Let

$$
E_{n}(f, G)_{M, \omega}:=\inf _{P \in \mathcal{P}_{n}}\|f-P\|_{L_{M}(\Gamma, \omega)}
$$

be the best approximation to $f \in E_{M}(G, \omega)$ in the class $\mathcal{P}_{n}$ of algebraic polynomials of degree not greater than $n$.

When $r=1$ and $\Gamma$ is a Carleson curve, some direct theorems of the approximation theory in the Smirnov-Orlicz and Orlicz classes are given in [8], 
[9]. One direct theorem in the Smirnov-Orlicz classes $E_{M}(G)$, defined on the domains with a Dini-smooth boundary, is obtained in [15]. The inverse problems of approximation theory in these domains have been investigated by $\mathrm{V}$. M. Kokilashvili [18]. Note that the modulus of smoothness used in these works are constructed by applying the usual shift $f_{0}\left(e^{i(t+h)}\right), h \in[0,2 \pi]$, for $f_{0}\left(e^{i t}\right)$.

In this work we prove some direct and inverse theorems in the weighted Smirnov-Orlicz classes. In particular, we obtain a constructive characterization of the generalized Lipschitz classes $\operatorname{Lip}^{*} \alpha(M, \omega), \alpha>0$. Since the usual shift, in general, is noninvariant in the weighted Orlicz classes, we use the modulus of smoothness $\Omega_{\Gamma, M, \omega}^{r}(f, \cdot)$, constructed with respect to the mean value operator $\sigma_{h}$

The main results of this work are the following.

Theorem 1. Let $G$ be a bounded simply connected domain with a Dinismooth boundary $\Gamma$ and let $L_{M}(\Gamma)$ be an Orlicz space with nontrivial indices $\alpha_{M}, \beta_{M}$ and $\omega \in A_{\frac{1}{\alpha_{M}}}(\Gamma) \cap A_{\frac{1}{\beta_{M}}}(\Gamma)$. If $f \in E_{M}(G, \omega)$, then for every natural number $n$,

$$
E_{n}(f, G)_{M, \omega} \leq c_{6} \Omega_{\Gamma, M, \omega}^{r}\left(f, \frac{1}{n+1}\right), \quad r=1,2,3, \ldots
$$

with some constant $c_{6}>0$ independent of $n$.

Theorem 2. Let $G$ be a bounded simply connected domain with a Dinismooth boundary $\Gamma$ and let $E_{M}(G, \omega)$ be a weighted Smirnov-Orlicz class with nontrivial indices $\alpha_{M}, \beta_{M}$. If $\omega \in A_{\frac{1}{\alpha_{M}}}(\Gamma) \cap A_{\frac{1}{\beta_{M}}}(\Gamma)$ and $f \in E_{M}(G, \omega)$, then

$$
\begin{array}{r}
\Omega_{\Gamma, M, \omega}^{r}\left(f, \frac{1}{n}\right) \leq \frac{c_{7}}{n^{2 r}}\left\{E_{0}(f, G)_{M, \omega}+\sum_{k=1}^{n} k^{2 r-1} E_{k}(f, G)_{M, \omega}\right\}, \\
r=1,2,3, \ldots,
\end{array}
$$

with some constant $c_{7}>0$ independent of $n$.

Corollary 1. Under the conditions of Theorem 2, if

$$
E_{n}(f, G)_{M, \omega}=\mathcal{O}\left(n^{-\alpha}\right), \quad \alpha>0, \quad n=1,2,3, \ldots,
$$

then

$$
\Omega_{\Gamma, M, \omega}^{r}(f, \delta)= \begin{cases}\mathcal{O}\left(\delta^{\alpha}\right) & ; r>\alpha / 2 \\ \mathcal{O}\left(\delta^{\alpha} \log \frac{1}{\delta}\right) & ; r=\alpha / 2 \\ \mathcal{O}\left(\delta^{2 r}\right) & ; r<\alpha / 2\end{cases}
$$

for $f \in L_{M}(\Gamma, \omega)$.

Definition 3. For $\alpha>0$ let $r:=\left[\frac{\alpha}{2}\right]+1$. The set of functions $f \in$ $E_{M}(G, \omega)$ such that

$$
\Omega_{\Gamma, M, \omega}^{r}(f, \delta)=\mathcal{O}\left(\delta^{\alpha}\right), \quad \delta>0
$$

is called the generalized Lipschitz class $\operatorname{Lip}^{*} \alpha(M, \omega)$. 
According to Corollary 1 we have the following.

Corollary 2. Under the conditions of Theorem 2, if

$$
E_{n}(f, G)_{M, \omega}=\mathcal{O}\left(n^{-\alpha}\right), \quad \alpha>0, \quad n=1,2,3, \ldots,
$$

then $f \in \operatorname{Lip}^{*} \alpha(M, \omega)$.

Theorem 1 and Corollary 2 imply the following.

Theorem 3. If $\alpha>0$, then under the conditions of Theorem 2, the following conditions are equivalent:

(a) $f \in \operatorname{Lip}^{*} \alpha(M, \omega)$

(b) $E_{n}(f)=\mathcal{O}\left(n^{-\alpha}\right), \quad n=1,2,3, \ldots$

In the case of weighted Smirnov classes $E^{p}(G, \omega)$ the analogues results are proved in the papers [11], [13].

Throughout this work by $c, c_{1}, c_{2}, \ldots$, we denote the constants which are different in different places.

\section{Auxiliary results}

Let $\Gamma$ be a rectifiable Jordan curve, $f \in L^{1}(\Gamma)$ and let

$$
\left(S_{\Gamma} f\right)(t):=\lim _{\varepsilon \rightarrow 0} \frac{1}{2 \pi i} \int_{\Gamma \backslash \Gamma(t, \epsilon)} \frac{f(\varsigma)}{\varsigma-t} d \varsigma, \quad t \in \Gamma
$$

be Cauchy's singular integral of $f$. The linear operator $S_{\Gamma}: f \rightarrow S_{\Gamma} f$ is called the Cauchy singular operator.

If one of the functions $f^{+}$or $f^{-}$has the non-tangential limits a. e. on $\Gamma$, then $S_{\Gamma} f(z)$ exist a. e. on $\Gamma$ and also the other one has non-tangential limits a. e. on $\Gamma$. Conversely, if $S_{\Gamma} f(z)$ exist a. e. on $\Gamma$, then both functions $f^{+}$and $f^{-}$have non-tangential limits a. e. on $\Gamma$. In both cases, the formulae

$$
\begin{aligned}
& f^{+}(z)=\left(S_{\Gamma} f\right)(z)+f(z) / 2, \\
& f^{-}(z)=\left(S_{\Gamma} f\right)(z)-f(z) / 2,
\end{aligned}
$$

and hence

$$
f=f^{+}-f^{-}
$$

holds a. e. on $\Gamma$ (see, e.g., [7, p. 431]).

Lemma 1. Let $0<\alpha_{M}, \beta_{M}<1, \omega \in A_{\frac{1}{\alpha_{M}}}(\Gamma) \cap A_{\frac{1}{\beta_{M}}}(\Gamma)$ and $f \in$ $L_{M}(\Gamma, \omega)$. Then $f^{+} \in E_{M}(G, \omega)$ and $f^{-} \in E_{M}\left(G^{-}, \omega\right)$. 
Proof. Let $f \in L_{M}(\Gamma, \omega)$. By [3, p. 58, Th. 2.31] there exist $p, q \in(1, \infty)$ such that $1<p<1 / \beta_{M} \leq 1 / \alpha_{M}<q<\infty$, and $\omega \in A_{p}(\Gamma) \cap A_{q}(\Gamma)$. Then [16, Th. 2.5] we have

$$
L^{q}(\Gamma) \subset L_{M}(\Gamma) \subset L^{p}(\Gamma),
$$

where the inclusion maps being continuous, and therefore $f \in L^{p}(\Gamma, \omega)$. Now using Lemmas 2 and 3 of [11] we get

$$
f^{+} \in E^{1}(G) \text { and } f^{-} \in E^{1}\left(G^{-}\right) .
$$

Hence, using the relations (2.1) which hold a. e. on $\Gamma$, and the boundedness of the singular operator $S_{\Gamma}$ in weighted Orlicz spaces $[17$, Th. 4.5], we conclude that

$$
f^{+} \in L_{M}(\Gamma, \omega), \quad f^{-} \in L_{M}(\Gamma, \omega)
$$

and the assertion follows.

Lemma 2. Let $0<\alpha_{M}, \beta_{M}<1, \omega \in A_{\frac{1}{\alpha_{M}}}(\mathbb{T}) \cap A_{\frac{1}{\beta_{M}}}(\mathbb{T})$ and $g \in$ $E_{M}(\mathbb{D}, \omega)$. If $\sum_{k=0}^{n} \alpha_{k} w^{k}$ is the nth partial sum of the Taylor series of the function $g$ at the origin, then there exists a constant $c_{8}>0$ such that

$$
\left\|g(w)-\sum_{k=0}^{n} \alpha_{k} w^{k}\right\|_{L_{M}(\mathbb{T}, \omega)} \leq c_{8} \Omega_{M, \omega}^{r}\left(g, \frac{1}{n+1}\right)
$$

for every natural number $n$.

This result was proved in [14, Theorem 3].

The Faber polynomials $\Phi_{k}(z), k=0,1,2,3, \ldots$, associated with $G \cup \Gamma$, are defined through the expansion

$$
\frac{\psi^{\prime}(w)}{\psi(w)-z}=\sum_{k=0}^{\infty} \frac{\Phi_{k}(z)}{w^{k+1}}, \quad z \in G, \quad w \in \mathbb{D}^{-},
$$

and the equalities

$$
\begin{gathered}
\Phi_{k}(z)=\frac{1}{2 \pi i} \int_{\mathbb{T}} \frac{w^{k} \psi^{\prime}(w)}{\psi(w)-z} d w, \quad z \in G, \\
\Phi_{k}(z)=\varphi^{k}(z)+\frac{1}{2 \pi i} \int_{\Gamma} \frac{\varphi^{k}(\varsigma)}{\varsigma-z} d \varsigma, \quad z \in G^{-},
\end{gathered}
$$

hold [23, p. 34].

If $f \in E_{M}(G, \omega)$, then by definition $f \in E^{1}(G)$ and hence

$$
\begin{aligned}
f(z) & =\frac{1}{2 \pi i} \int_{\Gamma} \frac{f(\varsigma)}{\varsigma-z} d \varsigma \\
& =\frac{1}{2 \pi i} \int_{\mathbb{T}} f(\psi(w)) \frac{\psi^{\prime}(w)}{\psi(w)-z} d w, \quad z \in G .
\end{aligned}
$$


Here, taking the relation (2.2) into account, we have

$$
f(z) \sim \sum_{k=0}^{\infty} a_{k} \Phi_{k}(z), \quad z \in G
$$

where

$$
a_{k}:=a_{k}(f):=\frac{1}{2 \pi i} \int_{\mathbb{T}} \frac{f(\psi(w))}{w^{k+1}} d w, \quad k=0,1,2, \ldots
$$

This series is called the Faber series of $f \in E_{M}(G, \omega)$ and the values $a_{k}$, $k=0,1,2, \ldots$ are called the Faber coefficients of $f$. Let $S_{n}(f, \cdot):=\sum_{k=0}^{n} a_{k} \Phi_{k}$ be the $n$th partial sum of the Faber expansion of the function $f \in E_{M}(G, \omega)$.

Let $\mathcal{P}:=\{$ all polynomials (with no restriction on the degree) $\}, \mathcal{P}(\mathbb{D}):=$ $\{$ traces of all members of $\mathcal{P}$ on $\mathbb{D}\}$ and let

$$
T(P)(z):=\frac{1}{2 \pi i} \int_{\mathbb{T}} \frac{P(w) \psi^{\prime}(w)}{\psi(w)-z} d w, \quad z \in G
$$

be an operator $T$ defined on $\mathcal{P}(\mathbb{D})$.

Then by $(2.3)$

$$
T\left(\sum_{k=0}^{n} b_{k} w^{k}\right)=\sum_{k=0}^{n} b_{k} \Phi_{k}(z), \quad z \in G .
$$

If $z^{\prime} \in G$, then

$$
\begin{aligned}
T(P)\left(z^{\prime}\right) & =\frac{1}{2 \pi i} \int_{\mathbb{T}} \frac{P(w) \psi^{\prime}(w)}{\psi(w)-z^{\prime}} d w=\frac{1}{2 \pi i} \int_{\Gamma} \frac{(P \circ \varphi)(\varsigma)}{\varsigma-z^{\prime}} d \varsigma \\
& =(P \circ \varphi)^{+}\left(z^{\prime}\right),
\end{aligned}
$$

which by (2.1) implies that

$$
T(P)(z)=S_{\Gamma}(P \circ \varphi)(z)+\frac{1}{2}(P \circ \varphi)(z)
$$

a. e. on $\Gamma$.

As in the proof of Lemma 1, there exist $p, q \in(1, \infty)$ such that $1<p<$ $1 / \beta_{M} \leq 1 / \alpha_{M}<q<\infty, \omega \in A_{p}(\Gamma) \cap A_{q}(\Gamma)$ and the inclusions

$$
L^{q}(\Gamma) \subset L_{M}(\Gamma) \subset L^{p}(\Gamma)
$$

hold. Then $P \circ \varphi \in L^{q}(\Gamma, \omega)$, for any polynomial $P$, and hence $P \circ \varphi \in$ $L_{M}(\Gamma, \omega)$. Since $S_{\Gamma}$ is bounded [17, Th. 4.5] in $L_{M}(\Gamma, \omega)$, from (2.5) we have that $T(P) \in L_{M}(\Gamma, \omega)$ for every $P \in \mathcal{P}(\mathbb{D})$. The property $T(P) \in E^{1}(G)$ can be obtained from continuity of $P \circ \varphi$. Hence we obtain $T(P) \in E_{M}(G, \omega)$ for every $P \in \mathcal{P}(\mathbb{D})$.

Therefore, we get the following result. 
Lemma 3. If $\Gamma$ is a Dini-smooth curve, $0<\alpha_{M}, \beta_{M}<1$ and $\omega \in$ $A_{\frac{1}{\alpha_{M}}}(\Gamma) \cap A_{\frac{1}{\beta_{M}}}(\Gamma)$, then the linear operator

$$
T: \mathcal{P}(\mathbb{D}) \rightarrow E_{M}(G, \omega)
$$

is bounded.

Extending the operator $T$ from $\mathcal{P}(\mathbb{D})$ to the space $E_{M}\left(\mathbb{D}, \omega_{0}\right)$ as a linear and bounded operator, for the extension $T: E_{M}\left(\mathbb{D}, \omega_{0}\right) \rightarrow E_{M}(G, \omega)$, we have the representation

$$
T(g)(z):=\frac{1}{2 \pi i} \int_{\mathbb{T}} \frac{g(w) \psi^{\prime}(w)}{\psi(w)-z} d w, \quad z \in G, \quad g \in E_{M}\left(\mathbb{D}, \omega_{0}\right) .
$$

Theorem 4. If $\Gamma$ is a Dini-smooth curve, $0<\alpha_{M}, \beta_{M}<1$ and $\omega \in$ $A_{\frac{1}{\alpha_{M}}}(\Gamma) \cap A_{\frac{1}{\beta_{M}}}(\Gamma)$, then the operator

$$
T: E_{M}\left(\mathbb{D}, \omega_{0}\right) \rightarrow E_{M}(G, \omega)
$$

is one-to-one and onto.

Proof. Let $g \in E_{M}\left(\mathbb{D}, \omega_{0}\right)$ with the Taylor expansion

$$
g(w):=\sum_{k=0}^{\infty} \alpha_{k} w^{k}, \quad w \in \mathbb{D}
$$

It is easily seen that if $\Gamma$ is Dini-smooth, then the conditions $\omega \in A_{\frac{1}{\alpha_{M}}}(\Gamma)$, $\omega_{0} \in A_{\frac{1}{\alpha_{M}}}(\mathbb{T})$ and also $\omega \in A_{\frac{1}{\beta_{M}}}(\Gamma), \omega_{0} \in A_{\frac{1}{\beta_{M}}}(\mathbb{T})$ are equivalent. Since $\omega_{0} \in A_{\frac{1}{\alpha_{M}}}(\mathbb{T}) \cap A_{\frac{1}{\beta_{M}}}(\mathbb{T})$, by the proof of Theorem 4.5 of [17] there exist $p, q \in(1, \infty)$ such that

$$
1<p<1 / \beta_{M} \leq 1 / \alpha_{M}<q<\infty \text { and } \omega_{0} \in A_{p}(\mathbb{T}) \cap A_{q}(\mathbb{T})
$$

and then, by [16, Th. 2.5],

$$
L^{q}(\mathbb{T}) \subset L_{M}(\mathbb{T}) \subset L^{p}(\mathbb{T}),
$$

where inclusion maps being continuous.

Let $g_{r}(w):=g(r w), 0<r<1$. Since $g \in E^{1}(\mathbb{D})$ is the Poisson integral of its boundary function [5, p. 41], using [21, Th. 10] and Boyd interpolation theorem [2], we get

$$
\left\|g_{r}-g\right\|_{L_{M}\left(\mathbb{T}, \omega_{0}\right)}=\left\|g\left(r e^{i \theta}\right)-g\left(e^{i \theta}\right)\right\|_{L_{M}\left([0,2 \pi], \omega_{0}\right)} \rightarrow 0, \quad \text { as } r \rightarrow 1^{-} .
$$


Therefore, the boundedness of the operator $T$ implies that

$$
\left\|T\left(g_{r}\right)-T(g)\right\|_{L_{M}(\Gamma, \omega)} \rightarrow 0, \quad \text { as } r \rightarrow 1^{-} .
$$

Since the series $\sum_{k=0}^{\infty} \alpha_{k} w^{k}$ is uniformly convergent for $|w|=r<1$, the series $\sum_{k=0}^{\infty} \alpha_{k} r^{k} w^{k}$ is uniformly convergent on $\mathbb{T}$, and hence

$$
\begin{aligned}
T\left(g_{r}\right)\left(z^{\prime}\right) & =\frac{1}{2 \pi i} \int_{\mathbb{T}} \frac{g_{r}(w) \psi^{\prime}(w)}{\psi(w)-z^{\prime}} d w=\sum_{m=0}^{\infty} \alpha_{m} r^{m} \frac{1}{2 \pi i} \int_{\mathbb{T}} \frac{w^{m} \psi^{\prime}(w)}{\psi(w)-z^{\prime}} d w \\
& =\sum_{m=0}^{\infty} \alpha_{m} r^{m} \Phi_{m}\left(z^{\prime}\right), \quad z^{\prime} \in G .
\end{aligned}
$$

Now, taking the limit as $z^{\prime} \rightarrow z \in \Gamma$ along all non-tangential paths inside $\Gamma$, we obtain

$$
T\left(g_{r}\right)(z)=\sum_{m=0}^{\infty} \alpha_{m} r^{m} \Phi_{m}(z), \quad z \in \Gamma .
$$

From the last equality and Lemma 3 of $[6$, p. 43] for the Faber coefficients $a_{k}\left(T\left(g_{r}\right)\right)$ we have

$$
\begin{aligned}
a_{k}\left(T\left(g_{r}\right)\right) & =\frac{1}{2 \pi i} \int_{\mathbb{T}} \frac{T\left(g_{r}\right)(\psi(w))}{w^{k+1}} d w \\
& =\frac{1}{2 \pi i} \int_{\mathbb{T}} \frac{\sum_{m=0}^{\infty} \alpha_{m} r^{m} \Phi_{m}(\psi(w))}{w^{k+1}} d w \\
& =\sum_{m=0}^{\infty} \alpha_{m} r^{m} \frac{1}{2 \pi i} \int_{\mathbb{T}} \frac{\Phi_{m}(\psi(w))}{w^{k+1}} d w=\alpha_{k} r^{k}
\end{aligned}
$$

and therefore

$$
a_{k}\left(T\left(g_{r}\right)\right) \rightarrow \alpha_{k}, \quad \text { as } r \rightarrow 1^{-} \text {. }
$$

Now applying (1.1), Hölder's inequality and Theorem 2.1 of [17], respectively, we obtain 


$$
\begin{aligned}
\left|a_{k}\left(T\left(g_{r}\right)\right)-a_{k}(T(g))\right| & =\left|\frac{1}{2 \pi i} \int_{\mathbb{T}} \frac{\left[T\left(g_{r}\right)-T(g)\right](\psi(w))}{w^{k+1}} d w\right| \\
& \leq \frac{1}{2 \pi} \int_{\mathbb{T}}\left|\left[T\left(g_{r}\right)-T(g)\right](\psi(w))\right||d w| \\
& =\frac{1}{2 \pi} \int_{\Gamma}\left|\left[T\left(g_{r}\right)-T(g)\right](z)\right|\left|\varphi^{\prime}(z)\right||d z| \\
& \leq \frac{c_{11}}{2 \pi} \int_{\Gamma}\left|\left[T\left(g_{r}\right)-T(g)\right](z)\right||d z| \\
& =\frac{c_{11}}{2 \pi} \int_{\Gamma}\left|\left[T\left(g_{r}\right)-T(g)\right](z)\right| \omega(z) \omega^{-1}(z)|d z| \\
& \leq \frac{c_{11}}{2 \pi}\left\|\left(T\left(g_{r}\right)-T(g)\right) \omega(z)\right\|_{L_{M}(\Gamma)}\left\|\omega^{-1}(\cdot)\right\|_{L_{N}(\Gamma)} \\
& \leq \frac{c_{12}}{2 \pi}\left\|T\left(g_{r}\right)-T(g)\right\|_{L_{M}(\Gamma, \omega)} .
\end{aligned}
$$

From the last inequality and (2.6) we get

$$
a_{k}\left(T\left(g_{r}\right)\right) \rightarrow a_{k}(T(g)), \quad \text { as } r \rightarrow 1^{-},
$$

and then by $(2.7) a_{k}(T(g))=\alpha_{k}, k=0,1,2, \ldots$ If $T(g)=0$, then $\alpha_{k}=$ $a_{k}(T(g))=0, k=0,1,2, \ldots$, and therefore $g=0$. This means that the operator $T$ is one-to-one.

Now we take a function $f \in E_{M}(G, \omega)$ and consider the function $f_{0}=$ $f \circ \psi \in L_{M}\left(\mathbb{T}, \omega_{0}\right)$. The Cauchy type integral

$$
\frac{1}{2 \pi i} \int_{\mathbb{T}} \frac{f_{0}(\tau)}{\tau-w} d \tau
$$

represents analytic functions $f_{0}^{+}$and $f_{0}^{-}$in $\mathbb{D}$ and $\mathbb{D}^{-}$, respectively. Since $\omega_{0} \in A_{\frac{1}{\alpha_{M}}}(\mathbb{T}) \cap A_{\frac{1}{\beta_{M}}}(\mathbb{T})$, by Lemma 1, we have

$$
f_{0}^{+} \in E_{M}\left(\mathbb{D}, \omega_{0}\right) \text { and } f_{0}^{-} \in E_{M}\left(\mathbb{D}^{-}, \omega_{0}\right),
$$

and for the non-tangential boundary values we get

$$
\begin{aligned}
& f_{0}^{+}(w)=S_{\mathbb{T}}\left(f_{0}\right)(w)+\frac{1}{2} f_{0}(w), \\
& f_{0}^{-}(w)=S_{\mathbb{T}}\left(f_{0}\right)(w)-\frac{1}{2} f_{0}(w) .
\end{aligned}
$$

Therefore

$$
f_{0}(w)=f_{0}^{+}(w)-f_{0}^{-}(w)
$$


holds a. e. on $\mathbb{T}$ and $f_{0}^{-}(\infty)=0$. For the Faber coefficients $a_{k}$ of $f$ we get

$$
\begin{aligned}
a_{k} & =\frac{1}{2 \pi i} \int_{\mathbb{T}} \frac{f_{0}(w)}{w^{k+1}} d w \\
& =\frac{1}{2 \pi i} \int_{\mathbb{T}} \frac{f_{0}^{+}(w)}{w^{k+1}} d w-\frac{1}{2 \pi i} \int_{\mathbb{T}} \frac{f_{0}^{-}(w)}{w^{k+1}} d w .
\end{aligned}
$$

Since the function $f_{0}^{-}$belongs to $E^{1}\left(\mathbb{D}^{-}\right)$, the second integral vanishes and hence the values $\left\{a_{k}\right\}_{k=0}^{\infty}$ also become the Taylor coefficients of the function $f_{0}^{+}$at the origin, namely,

$$
f_{0}^{+}(w)=\sum_{k=0}^{\infty} a_{k} w^{k}, \quad w \in \mathbb{D} .
$$

From the first part of the proof we get

$$
T\left(f_{0}^{+}\right) \backsim \sum_{k=0}^{\infty} a_{k} \Phi_{k} .
$$

Since there is no two different functions in $E_{M}(G, \omega)$ that have the same Faber coefficients [1], we conclude that $T\left(f_{0}^{+}\right)=f$. Therefore, the operator $T$ is onto.

\section{Proofs of main results}

Proof of Theorem 1 . Let $f \in E_{M}(G, \omega)$. Then $f_{0} \in L_{M}\left(\mathbb{T}, \omega_{0}\right)$. According to $(2.8)$

$$
f(\varsigma)=f_{0}^{+}(\varphi(\varsigma))-f_{0}^{-}(\varphi(\varsigma))
$$

a. e. on $\Gamma$ and

$$
\int_{\Gamma} \frac{f(\varsigma)}{\varsigma-z^{\prime}} d \varsigma=0, \quad z^{\prime} \in G^{-}
$$

because $f \in E^{1}(G)$.

Now let $z^{\prime} \in G^{-}$. Using (2.4) we have

$$
\begin{aligned}
\sum_{k=0}^{n} a_{k} \Phi_{k}\left(z^{\prime}\right)= & \sum_{k=0}^{n} a_{k} \varphi^{k}\left(z^{\prime}\right)+\frac{1}{2 \pi i} \int_{\Gamma} \frac{\sum_{k=0}^{n} a_{k} \varphi^{k}(\varsigma)}{\varsigma-z^{\prime}} d \varsigma \\
= & \sum_{k=0}^{n} a_{k} \varphi^{k}\left(z^{\prime}\right)+\frac{1}{2 \pi i} \int_{\Gamma} \frac{\sum_{k=0}^{n} a_{k} \varphi^{k}(\varsigma)}{\varsigma-z^{\prime}} d \varsigma-\frac{1}{2 \pi i} \int_{\Gamma} \frac{f(\varsigma)}{\varsigma-z^{\prime}} d \varsigma \\
= & \sum_{k=0}^{n} a_{k} \varphi^{k}\left(z^{\prime}\right)+\frac{1}{2 \pi i} \int_{\Gamma} \frac{\sum_{k=0}^{n} a_{k} \varphi^{k}(\varsigma)}{\varsigma-z^{\prime}} d \varsigma \\
& -\frac{1}{2 \pi i} \int_{\Gamma} \frac{f_{0}^{+}(\varphi(\varsigma))}{\varsigma-z^{\prime}} d \varsigma+\frac{1}{2 \pi i} \int_{\Gamma} \frac{f_{0}^{-}(\varphi(\varsigma))}{\varsigma-z^{\prime}} d \varsigma .
\end{aligned}
$$


Since

$$
\frac{1}{2 \pi i} \int_{\Gamma} \frac{f_{0}^{-}(\varphi(\varsigma))}{\varsigma-z^{\prime}} d \varsigma=-f_{0}^{-}\left(\varphi\left(z^{\prime}\right)\right)
$$

we get

$$
\begin{aligned}
\sum_{k=0}^{n} a_{k} \Phi_{k}\left(z^{\prime}\right)= & \frac{1}{2 \pi i} \int_{\Gamma} \frac{\sum_{k=0}^{n} a_{k} \varphi^{k}(\varsigma)-f_{0}^{+}(\varphi(\varsigma))}{\varsigma-z^{\prime}} d \varsigma \\
& +\sum_{k=0}^{n} a_{k} \varphi^{k}\left(z^{\prime}\right)-f_{0}^{-}\left(\varphi\left(z^{\prime}\right)\right) .
\end{aligned}
$$

Hence, taking the limit as $z^{\prime} \rightarrow z$ along all non-tangential paths outside $\Gamma$, we obtain

$$
\begin{aligned}
\sum_{k=0}^{n} a_{k} \Phi_{k}(z)= & -\frac{1}{2}\left(\sum_{k=0}^{n} a_{k} \varphi^{k}(z)-f_{0}^{+}(\varphi(z))\right)+S_{\Gamma}\left[\sum_{k=0}^{n} a_{k} \varphi^{k}-\left(f_{0}^{+} \circ \varphi\right)\right] \\
& +\sum_{k=0}^{n} a_{k} \varphi^{k}(z)-f_{0}^{-}(\varphi(z)) \\
= & \frac{1}{2}\left(\sum_{k=0}^{n} a_{k} \varphi^{k}(z)-f_{0}^{+}(\varphi(z))\right)+\left[f_{0}^{+}(\varphi(z))-f_{0}^{-}(\varphi(z))\right] \\
& +S_{\Gamma}\left[\sum_{k=0}^{n} a_{k} \varphi^{k}-\left(f_{0}^{+} \circ \varphi\right)\right]
\end{aligned}
$$

a. e. on $\Gamma$. Using (3.1), (1.1), Minkowski's inequality and the boundedness of $S_{\Gamma}$ we get

$$
\begin{aligned}
& \left\|f-S_{n}(f, \cdot)\right\|_{L_{M}(\Gamma, \omega)} \\
& =\left\|\frac{1}{2}\left(\sum_{k=0}^{n} a_{k} \varphi^{k}(z)-f_{0}^{+}(\varphi(z))\right)+S_{\Gamma}\left[\sum_{k=0}^{n} a_{k} \varphi^{k}-\left(f_{0}^{+} \circ \varphi\right)\right]\right\|_{L_{M}(\Gamma, \omega)} \\
& \leq c_{13}\left\|\sum_{k=0}^{n} a_{k} \varphi^{k}(z)-f_{0}^{+}(\varphi(z))\right\|_{L_{M}(\Gamma, \omega)} \leq c_{14}\left\|f_{0}^{+}(w)-\sum_{k=0}^{n} a_{k} w^{k}\right\|_{L_{M}\left(\mathbb{T}, \omega_{0}\right)} .
\end{aligned}
$$

On the other hand, from the proof of Theorem 4 we know that the Faber coefficients of the function $f$ and the Taylor coefficients of the function $f_{0}^{+}$at the origin are the same. Then taking Lemma 2 into account, we conclude that

$$
E_{n}(f, G)_{M, \omega} \leq\left\|f-S_{n}(f, \cdot)\right\|_{L_{M}(\Gamma, \omega)} \leq c_{15} \Omega_{\Gamma, M, \omega}^{r}\left(f, \frac{1}{n+1}\right) .
$$


Proof of Theorem 2. Let $f \in E_{M}(G, \omega)$. Then by the proof of Theorem 4 we have $T\left(f_{0}^{+}\right)=f$. Since the operator $T: E_{M}\left(\mathbb{D}, \omega_{0}\right) \rightarrow E_{M}(G, \omega)$ is linear, bounded, one-to-one and onto, the operator $T^{-1}: E_{M}(G, \omega) \rightarrow E_{M}\left(\mathbb{D}, \omega_{0}\right)$ is linear and bounded. We take a $p_{n}^{*} \in \mathcal{P}_{n}$ as the best approximating algebraic polynomial to $f$ in $E_{M}(G, \omega)$, i.e.,

$$
E_{n}(f, G)_{M, \omega}=\left\|f-p_{n}^{*}\right\|_{L_{M}(\Gamma, \omega)} .
$$

(There exists such a unique polynomial $p_{n}^{*}$ of $\mathcal{P}_{n}$, see, for example, [4, p. 59]). Then $T^{-1}\left(p_{n}^{*}\right) \in \mathcal{P}_{n}(\mathbb{D})$ and therefore

$$
\begin{aligned}
E_{n}\left(f_{0}^{+}, \mathbb{D}\right)_{M, \omega_{0}} & \leq\left\|f_{0}^{+}-T^{-1}\left(p_{n}^{*}\right)\right\|_{L_{M}\left(\mathbb{T}, \omega_{0}\right)}=\left\|T^{-1}(f)-T^{-1}\left(p_{n}^{*}\right)\right\|_{L_{M}\left(\mathbb{T}, \omega_{0}\right)} \\
& =\left\|T^{-1}\left(f-p_{n}^{*}\right)\right\|_{L_{M}\left(\mathbb{T}, \omega_{0}\right)} \leq\left\|T^{-1}\right\|\left\|f-p_{n}^{*}\right\|_{L_{M}(\Gamma, \omega)} \\
& =\left\|T^{-1}\right\| E_{n}(f, G)_{M, \omega},
\end{aligned}
$$

because the operator $T^{-1}$ is bounded.

On the other hand, from [14] we have

$$
\Omega_{M, \omega_{0}}^{r}\left(f_{0}^{+}, \frac{1}{n}\right) \leq \frac{c_{16}}{n^{2 r}}\left\{E_{0}\left(f_{0}^{+}, \mathbb{D}\right)_{M, \omega_{0}}+\sum_{k=1}^{n} k^{2 r-1} E_{k}\left(f_{0}^{+}, \mathbb{D}\right)_{M, \omega_{0}}\right\}
$$

$r=1,2, \ldots$

The last inequality and (3.2) imply that

$$
\begin{aligned}
\Omega_{\Gamma, M, \omega}^{r}\left(f, \frac{1}{n}\right) & =\Omega_{M, \omega_{0}}^{r}\left(f_{0}^{+}, \frac{1}{n}\right) \\
& \leq \frac{c_{16}}{n^{2 r}}\left\{E_{0}\left(f_{0}^{+}, \mathbb{D}\right)_{M, \omega_{0}}+\sum_{k=1}^{n} k^{2 r-1} E_{k}\left(f_{0}^{+}, \mathbb{D}\right)_{M, \omega_{0}}\right\} \\
& \leq \frac{c_{16}\left\|T^{-1}\right\|}{n^{2 r}}\left\{E_{0}(f, G)_{M, \omega}+\sum_{k=1}^{n} k^{2 r-1} E_{k}(f, G)_{M, \omega}\right\},
\end{aligned}
$$

$r=1,2, \ldots$

Acknowledgements. The authors are indebted to Dr. Ali Guven for constructive discussions and also to referees for valuable suggestions.

BALIKESIR UNIVERSITY

FACUlTy OF ARTS AND SCIENCES

Department OF MATHEMATiCS

10145, BALIKESIR, TURKEY

e-mail: mdaniyal@balikesir.edu.tr

rakgun@balikesir.edu.tr 


\section{References}

[1] J-E. Andersson, On the degree of polynomial approximation in $E^{p}(D), \mathrm{J}$. Approx. Theory 19 (1977), 61-68.

[2] D. W. Boyd, Spaces between a pair of reflexive Lebesgue spaces, Proc. Amer. Math. Soc. 18 (1967), 215-219.

[3] A. Böttcher and Yu. I. Karlovich, Carleson curves, Muckenhoupt weights, and Toeplitz operators, Progress in Mathematics 154, Birkhäuser Verlag, 1997.

[4] R. A. DeVore and G. G. Lorentz, Constructive approximation, SpringerVerlag, 1993.

[5] P. L. Duren, Theory of $H^{p}$ spaces, Academic Press, 1970.

[6] D. Gaier, Lectures on complex approximation, Birkhäuser, 1987.

[7] G. M. Goluzin, Geometric theory of functions of a complex variable, Transl. Math. Monogr. 26, R. I., AMS, Providence, 1969.

[8] A. Guven and D. M. Israfilov, Polynomial approximation in SmirnovOrlicz classes, Comput. Methods Funct. Theory 2-2 (2002), 509-517.

[9] _ Rational approximation in Orlicz spaces on Carleson curves, Bull. Belg. Math. Soc. Simon Stevin 12-2 (2005), 223-234.

[10] E. A. Haciyeva, Investigation the properties of the functions with quasimonotone Fourier coefficients in generalized Nikolsky-Besov spaces, Author's summary of candidates dissertation, Tbilisi, (Russian), 1986.

[11] D. M. Israfilov, Approximation by p-Faber polynomials in the weighted Smirnov class $E^{p}(G, \omega)$ and the Bieberbach polynomials, Constr. Approx. 17-3 (2001), 335-351.

[12] _ Approximation by p-Faber-Laurent rational functions in the weighted Lebesgue spaces, Czechoslovak Math. J. 54-3 (2004), 751-765.

[13] D. M. Israfilov and A. Guven, Approximation in weighted Smirnov classes, East J. Approx. 11-1 (2005), 91-102.

[14] _ Approximation by trigonometric polynomials in weighted Orlicz spaces, Studia Math. 174-2 (2006), 147-168.

[15] D. M. Israfilov, B. Oktay and R. Akgun, Approximation in Smirnov-Orlicz classes, Glasnik Matematički 40-1 (2005), 87-102.

[16] A. Yu. Karlovich, Algebras of singular integral operators with piecewise coefficients on reflexive Orlicz spaces, Math. Nachr. 179 (1996), 187-222. 
[17] _ Algebras of singular integral operators with PC coefficients in rearrangement-invariant spaces with Muckenhoupt weights, J. Operator Theory 47 (2002), 303-323.

[18] V. M. Kokilashvili, On analytic functions of Smirnov-Orlicz classes, Studia Math. 31 (1968), 43-59.

[19] L. Maligranda, Indices and interpolation, Dissertationes Math. 234 (1985), $1-49$.

[20] W. Matuszewska and W. Orlicz, On certain properties of $\varphi$-functions, Bull. Acad. Polon. Sci., Ser. Math. Aster. et Phys. 8-7 (1960), 439-443.

[21] B. Muckenhoupt, Weighted norm inequalities for the Hardy maximal function, Trans. Amer. Math. Soc. 167 (1972), 207-226.

[22] Ch. Pommerenke, Boundary behavior of conformal maps, Springer-Verlag, 1992.

[23] P. K. Suetin, Series of Faber polynomials, Gordon and Breach, 1, Reading, 1998.

[24] S. E. Warschawski, Über das Ranverhalten der Ableitung der Abbildungsfunktion bei konformer Abbildung, Math. Z. 35 (1932), 321-456.

[25] M. Wehrens, Best approximation on the unit sphere in $\mathbb{R}^{n}$, Proc. Conf. Oberwolfach. August, 9-16, Basel e. a. pp. 233-245, Funct. Anal. Approx., 1981. 